\title{
DETECTION OF A RANDOM WALK SIGNAL IN THE REGIME OF LOW SIGNAL TO NOISE RATIO AND LONG OBSERVATION TIME
}

\author{
Michael Ting and Alfred O. Hero III \\ Electrical Engineering and Computer Science, University of Michigan \\ Ann Arbor, MI 48109-2122, USA \\ \{mting,hero\}@eecs.umich.edu
}

\begin{abstract}
This paper considers the detection of a Markov signal in additive white Gaussian noise (AWGN). Here, the Markov signal is taken to be a certain class of random walk processes. A closed form expression of the likelihood ratio (LR) is derived for a general Markov signal in AWGN. Then, under the conditions of low signal to noise ratio (SNR) and long observation time, necessary conditions are derived for the LR of the random walk to be approximated by a bank of filtered energy (FE) detectors, as well as by a single FE detector. The FE detector is an intuitive way to perform detection; however, it is not necessarily optimal. The results are applicable to the detection of an electron spin in a magnetic resonance force microscopy (MRFM) experiment.
\end{abstract}

\section{INTRODUCTION}

The detection of a discrete-time Markov signal in AWGN is widespread. Under the null hypothesis, the observations consist of AWGN, while in the other, they consist of a Markov signal and AWGN. In [1], the case when the Markov signal is a random telegraph process was considered. It was shown that the optimal likelihood ratio test (LRT) for the random telegraph model can be approximated by a FE detector under the following four conditions: low SNR, long observation time, symmetric transition probabilities, and small probability of transition between consecutive time samples. In this paper, an analogous result is derived when the Markov signal is taken to be a random walk. We derive necessary conditions for the LRT of a class of random walk processes to be approximated by a bank of FE detectors and by a single FE detector under the conditions of low SNR and long observation time.

The results of this paper are applicable to MRFM, where the detection of a single electron spin can be modelled as the binary hypothesis test considered above. Two models were proposed for the Markov signal that represents the electron spin: the random telegraph and random walk process. Current MRFM single-spin experiments occur in the regime of low SNR and long observation time $[1,2]$, which is precisely the regime that is considered in this paper. In addition, our results can be applied to [3], where the detection of random walks in capital markets is of interest. In [4], the authors construct a random walk model for the fluctuations in the sun's magnetic field. Our results can be applied to determine if the observations of the sun's magnetic field does indeed support a random walk model.

This work was supported in part by the DARPA Mosaic program under ARO contract DAAD19-02-C-0055 and by ARO MURI grant W911NF-051-0403.

\section{PROBLEM FORMULATION}

Let $X_{i}, i=0, \ldots, N-1$ denote a Markov signal with state space $\Psi=\left\{\psi_{1}, \ldots, \psi_{d}\right\}$, where $d$ is the number of possible values that $X_{i}$ can assume. Let $\mathbf{P}=\left(p_{j k}\right) \in \mathbb{R}^{d \times d}$ be the probability transition matrix associated with $X_{i}$ so that $p_{j k}=P\left(X_{i}=\psi_{k} \mid X_{i-1}=\right.$ $\left.\psi_{j}\right) \forall 1 \leq i \leq N-1$. Define $W_{i}$ to be AWGN noise with variance $\sigma^{2}$ and $y_{i}$ to be the observations for $i=0, \ldots, N-1$. Let $\underline{y} \triangleq\left[y_{0}, \ldots, y_{N-1}\right]^{T}$, where $(\cdot)^{T}$ is the transpose operator. The detection problem is to design a test between the two hypotheses

$$
\begin{aligned}
& H_{0}: y_{i}=w_{i}, \quad 0 \leq i \leq N-1 \\
& H_{1}: y_{i}=x_{i}+w_{i}, \quad 0 \leq i \leq N-1
\end{aligned}
$$

In this article, we consider the case when $X_{i}$ is a certain class of random walks. Specifically, we assume that the probability transition matrix $\mathbf{P}$ has the following properties: (A1) it is a tridiagonal matrix; (A2) $p_{j, j+1} p_{j+1, j}>0$ for $1 \leq j<d$; (A3) $p_{j j}=0$ for $1 \leq j \leq d$. Note that as $\mathbf{P}$ is a stochastic matrix, each $p_{j k}$ is a non-negative real and each row of $\mathbf{P}$ sums to 1 .

Let $f_{i}(Z)$ be the density of $Z$ induced under hypothesis $H_{i}, i=$ 0,1 . Similarly, let $E_{i}[\cdot]$ and $\operatorname{var}_{i}(\cdot)$ denote the expectation and variance respectively under hypothesis $H_{i}, i=0,1$. The definition of SNR used in this paper is the same as in [1], viz.,

$$
\begin{aligned}
\mathrm{SNR} \triangleq N\left\{-\frac{1}{2}\right. & \log \frac{2 \sqrt{\operatorname{var}_{0}\left(Y_{i}\right) \operatorname{var}_{1}\left(Y_{i}\right)}}{\operatorname{var}_{0}\left(Y_{i}\right)+\operatorname{var}_{1}\left(Y_{i}\right)} \\
& \left.+\frac{\left(E_{1}\left[Y_{i}\right]-E_{0}\left[Y_{i}\right]\right)^{2}}{4\left(\operatorname{var}_{0}\left(Y_{i}\right)+\operatorname{var}_{1}\left(Y_{i}\right)\right)}\right\},
\end{aligned}
$$

which is the $\alpha$-divergence of the densities $f_{1}(y)$ and $f_{0}(y)$ with $\alpha=$ $1 / 2$. When $Y_{i}$ is not wide-sense stationary, the SNR is a function of $i$. In this case, the SNR will be taken to be the steady-state value, i.e., $\mathrm{SNR} \triangleq \lim _{i \rightarrow \infty} \mathrm{SNR}_{i}$. The condition of low SNR will be taken to mean that $\left|\psi_{k} / \sigma\right| \ll 1$ for $1 \leq k \leq d$.

\section{DETECTION STRATEGIES}

\subsection{Unfiltered and filtered energy detector}

The filtered energy detector is an extension of the unfiltered energy detector. Consider the unfiltered energy detector: if the signal and noise are independent, which is the case for the detection problem considered here, the energy term should be higher under hypothesis $H_{1}$ than under hypothesis $H_{0}$. Now, if the signal $Y_{i}$ is lowpass, an intuitive way to improve detection performance is to pre-filter the observations $y_{i}$ with a lowpass filter. Most of the signal would be retained, but noise outside of the lowpass filter bandwidth would be 
suppressed. Effectively, the SNR is increased. We shall use the same first-order, single-pole lowpass filter (LPF) as in [1]. Let $h_{\mathrm{LP}}[i]$ be the impulse response of the filter with response

$$
H_{\mathrm{LP}}(z)=\frac{1-\alpha_{\mathrm{LP}}}{2} \frac{1+z^{-1}}{1-\alpha_{\mathrm{LP}} z^{-1}},
$$

where $\left|\alpha_{\mathrm{LP}}\right|<1$ is required for stability [5]. Let “ $*$ ” denote the convolution operator, so that $s=y * h$ is defined by $s_{i} \triangleq \sum_{n} y_{n} h_{i-n}$. The filtered and unfiltered energy detectors are given by

$$
\sum_{i}(y * h)_{i}^{2} \underset{H_{0}}{\stackrel{H_{1}}{\gtrless}} \eta
$$

where, in the former, $h[i]=h_{\mathrm{LP}}[i]$ and in the latter, $h[i]=\delta[i]$. Let $g$ be the FE statistic, i.e. the LHS of (4) with $h[i]=h_{\mathrm{LP}}[i]$, and $\tilde{y}_{i} \triangleq y_{i} / \sigma$ be the normalized observations. For sufficiently large $N$, it can be shown that

$$
g \approx C\left\{\sum_{j<k} \alpha_{\mathrm{LP}}^{k-j} \tilde{y}_{j} \tilde{y}_{k}+\frac{\alpha_{\mathrm{LP}}}{1+\alpha_{\mathrm{LP}}} \sum_{j} \tilde{y}_{j}^{2}\right\}
$$

where $C$ is a constant independent of the observations [1]. Note that $C$ plays no role in the performance of the FE detector.

\subsection{Likelihood ratio test}

The LRT is a most powerful (MP) test that satisfies the NeymanPearson criterion: it maximizes the probability of detection $\left(P_{D}\right)$ subject to a constraint on the probability of false alarm $\left(P_{F}\right)$ [6]. One can derive a closed-form expression of the LR for the binary hypothesis test problem of (1).

Several definitions are necessary before proceeding. We shall adopt notation and terminology that are consistent with [1,7]. Let $\pi_{k}, 1 \leq k \leq d$ be the initial probabilities of $X_{0}$, and $\underline{\pi} \triangleq$ $\left[\pi_{1}, \ldots, \pi_{d}\right]^{T}$. Define $\varphi\left(\theta ; \mu, \sigma^{2}\right) \triangleq \frac{1}{\sqrt{2 \pi} \sigma} \exp \left[-(\theta-\mu)^{2} / 2 \sigma^{2}\right]$. For $\underline{\theta} \in \mathbb{R}^{d}, \operatorname{diag}(\underline{\theta})$ is the $d$-by- $d$ matrix with $\underline{\theta}$ along its diagonal. For $0 \leq i \leq N-1$, let $y^{i} \triangleq\left[y_{0}, \ldots, y_{i}\right]^{T}$, and

$$
\begin{aligned}
\underline{n}_{i} & \triangleq\left[\varphi\left(y_{i} ; \psi_{1}, \sigma^{2}\right), \ldots, \varphi\left(y_{i} ; \psi_{d}, \sigma^{2}\right)\right]^{T} \\
\mathbf{H}^{(i)} & \triangleq \operatorname{diag}\left(\underline{n}_{i}\right) / \varphi\left(y_{i} ; 0, \sigma^{2}\right) .
\end{aligned}
$$

For $i \geq 1$, let

$$
\begin{gathered}
\boldsymbol{\Omega}^{(i)} \triangleq \operatorname{diag}\left(\underline{n}_{i}\right) / f_{1}\left(y_{i} \mid y^{i-1}\right) \\
l\left(y_{i} \mid y^{i-1}\right) \triangleq f_{1}\left(y_{i} \mid y^{i-1}\right) / f_{0}\left(y_{i} \mid y^{i-1}\right) \\
\underline{q}_{i} \triangleq\left[P\left(X_{i}=\psi_{1} \mid y^{i-1}\right), \ldots, P\left(X_{i}=\psi_{d} \mid y^{i-1}\right)\right]^{T}
\end{gathered}
$$

Eqn. (9) defines the transition likelihood ratio, and (10) defines the vector of prediction probabilities.

Proposition 1 The LR for the binary hypothesis test problem of (1) is

$$
L_{N}=\underline{\pi}^{T} \mathbf{H}^{(0)} \mathbf{P} \mathbf{H}^{(1)} \mathbf{P} \cdots \mathbf{H}^{(N-1)} \underline{1}
$$

where $\underline{1}=[1, \ldots, 1]^{T} \in \mathbb{R}^{d}$, and $\mathbf{P}$ is the probability transition matrix.

The result can be obtained by using $[1,(15)]$ and the fact that $\boldsymbol{\Omega}^{(i)}=\mathbf{H}^{(i)}\left(l\left(y_{i} \mid y^{i-1}\right)\right)^{-1}$ for $i \geq 1$. It can be shown that

$$
\underline{q}_{i}^{T}=\underline{q}_{i-1}^{T} \frac{\mathbf{H}^{(i-1)}}{l\left(y_{i-1} \mid y^{i-2}\right)} \mathbf{P}, i \geq 2
$$

The LR is $L_{N}=\prod_{i=1}^{N-1} l\left(y_{k} \mid y^{k-1}\right) \cdot L_{1}$, where $L_{1} \triangleq f_{1}\left(y_{0}\right) / f_{0}\left(y_{0}\right)$. Starting with

$$
\begin{aligned}
l\left(y_{N-1} \mid y^{N-2}\right) & =\underline{q}_{N-1}^{T} \underline{n}_{N-1}\left(\varphi\left(y_{N-1} ; 0, \sigma^{2}\right)\right)^{-1} \\
& =\underline{q}_{N-1}^{T} \mathbf{H}^{(N-1)} \underline{1}
\end{aligned}
$$

and repeatedly applying (12), one arrives at

$$
\prod_{i=1}^{N-1} l\left(y_{k} \mid y^{k-1}\right)=\underline{q}_{1}^{T} \mathbf{H}^{(1)} \mathbf{P} \mathbf{H}^{(2)} \ldots \mathbf{P} \mathbf{H}^{(N-1)} \underline{1}
$$

The result follows by using $L_{1} \underline{q}_{1}^{T}=\underline{\pi}^{T} \mathbf{H}^{(0)} \mathbf{P}$.

\subsection{Approximation to the LRT}

We are interested in deriving an approximation to the LRT of the random walk under the regime of low SNR and long observation time, i.e. large $N$. We shall begin with two propositions.

Proposition 2 Suppose $\mathbf{A}=\left(a_{j k}\right) \in \mathbb{R}^{d \times d}$ is a real tridiagonal matrix. If $a_{k k}=0$ for $1 \leq k \leq d$, then whenever $\lambda \in \mathbb{R}$ is an eigenvalue of $\mathbf{A}$, so is $(-\lambda)$.

Refer to [8]

Proposition 3 The probability transition matrix $\mathbf{P}$ that satisfies assumptions A1-A3 has the following properties:

B1. Let $\lambda_{1}, \ldots, \lambda_{d}$ be the eigenvalues of $\mathbf{P}$. Then, they can be ordered as $\lambda_{1}>\lambda_{2}>\ldots>\lambda_{d}$ with $\lambda_{k} \in \mathbb{R}$ for $1 \leq k \leq d$

B2. $\lambda_{k}+\lambda_{d+1-k}=0$ for $1 \leq k \leq d$

B3. $\lambda_{1}=1$

First, let us show B1. Now, the matrix $\mathbf{P}=\left(p_{j k}\right)$ for a random walk is tridiagonal. Since $p_{j, j+1} p_{j+1, j}>0$ for $1 \leq j<d$ by assumption A2, $\mathbf{P}$ has only real simple eigenvalues [9]. To show B2, apply Prop. 2. Finally, B3 follows from the fact that the spectral radius of a stochastic matrix is $1[10$, p. 3].

Consider $\mathbf{H}^{(i)}$, which is a diagonal matrix with the $(k, k)$-th entry equal to

$$
\frac{\varphi\left(y_{i} ; \psi_{k}, \sigma^{2}\right)}{\varphi\left(y_{i} ; 0, \sigma^{2}\right)}=\exp \left(\frac{2 y_{i} \psi_{k}-\psi_{k}^{2}}{2 \sigma^{2}}\right) \approx e^{-\frac{\psi_{k}^{2}}{2 \sigma^{2}}}\left(1+\frac{\psi_{k} y_{i}}{\sigma^{2}}\right),
$$

where the last statement is justified by using the low SNR assumption and the approximation $e^{\delta} \approx 1+\delta$ for small $|\delta|$. Use (15) in Prop. 1; then, expand (11). The result is that $L_{N} \approx L_{N, 1}+L_{N, 2 a}+$ $L_{N, 2 b}+$ higher order terms, where

- $L_{N, 1}$ is an expression that is linear in $y_{i}, 0 \leq i \leq N-1$. If $E_{1}\left[Y_{i}\right]=E_{0}\left[Y_{i}\right] \forall 0 \leq i \leq N-1$, then to the first moment, $L_{N, 1}$ does not play a role in the LRT.

- $L_{N, 2 a}$ is the effect of $y_{0}$ on the LR. When $N$ is large, we expect that the effect is negligible compared to $L_{N, 2 b}$.

- $L_{N, 2 b}$ consists of terms of the form $y_{j} y_{k}$ for $1 \leq j<k \leq$ $N-1$.

Let us examine $L_{N, 2 b}$ in further detail. Define the matrices $\mathbf{Q} \triangleq \mathbf{P} \cdot \operatorname{diag}\left(e^{-\psi_{1}^{2} / 2 \sigma^{2}}, \ldots, e^{-\psi_{d}^{2} / 2 \sigma^{2}}\right)$ and $\mathbf{R} \triangleq \mathbf{Q}$. $\operatorname{diag}\left(\psi_{1} / \sigma, \ldots, \psi_{d} / \sigma\right)$. It can be shown that $\mathbf{Q}$ satisfies $\mathbf{B} 1$ and B2 [8]. Let $\kappa_{1}, \ldots, \kappa_{d}$ be the eigenvalues of $\mathbf{Q}$, with $\kappa_{1}>\ldots>$ $\kappa_{d}$. Then, $\kappa_{1} \leq 1$ [8]. Let $\mathbf{U}_{Q}$ be the matrix that contains the 
eigenvectors of $\mathbf{Q}$, and $\boldsymbol{\Lambda}_{Q} \triangleq \operatorname{diag}\left(\kappa_{1}, \ldots, \kappa_{d}\right)$. $\mathbf{U}_{Q}$ is invertible since the eigenvalues $\kappa_{i}$ are all distinct. Define $\widetilde{\boldsymbol{\Lambda}}_{Q} \triangleq \kappa_{1}^{-1} \boldsymbol{\Lambda}_{Q}$ and $\widetilde{\mathbf{R}} \triangleq \mathbf{U}_{Q}^{-1} \mathbf{R} \mathbf{U}_{Q}$. Denote $\kappa_{k}^{\prime} \triangleq \kappa_{k} / \kappa_{1}$ for $1 \leq k \leq d$, so that $\widetilde{\boldsymbol{\Lambda}}_{Q}=\operatorname{diag}\left(\kappa_{1}^{\prime}, \ldots, \kappa_{d}^{\prime}\right)$, where $1=\kappa_{1}^{\prime}>\ldots>\kappa_{d}^{\prime}=-1 . L_{N, 2 b}$ can be expressed as

$$
\begin{aligned}
& L_{N, 2 b}=\frac{1}{\kappa_{1}^{2}} \sum_{1 \leq j<k \leq N-1} \tilde{y}_{j} \tilde{y}_{k} \underline{a}^{T} \mathbf{\Upsilon}[j, k] \underline{b} \\
& \text { where: } \boldsymbol{\Upsilon}[j, k] \triangleq \widetilde{\boldsymbol{\Lambda}}_{Q}^{j-1} \widetilde{\mathbf{R}} \widetilde{\boldsymbol{\Lambda}}_{Q}^{k-1-j} \widetilde{\mathbf{R}} \widetilde{\boldsymbol{\Lambda}}_{Q}^{N-1-k}
\end{aligned}
$$

and $\underline{a}, \underline{b}$ are independent of the observations. For $N$ large, most of

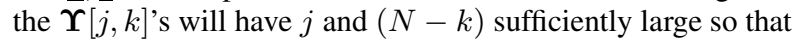

$$
\begin{aligned}
\widetilde{\boldsymbol{\Lambda}}_{Q}^{j-1} & \approx \operatorname{diag}\left(1,0, \ldots, 0,(-1)^{j-1}\right) \text { and } \\
\widetilde{\boldsymbol{\Lambda}}_{Q}^{N-1-k} & \approx \operatorname{diag}\left(1,0, \ldots, 0,(-1)^{N-1-k}\right)
\end{aligned}
$$

Let $\mathbf{J}_{j k}$ be a $d$-by- $d$ matrix with all zeros except for a 1 in the $(j, k)$ th position. Using (18),

$$
\begin{gathered}
\mathbf{\Upsilon}[j, k] \approx \mathbf{J}_{11} \widetilde{\mathbf{R}} \widetilde{\Lambda}_{Q}^{k-1-j} \widetilde{\mathbf{R}} \mathbf{J}_{11}+ \\
(-1)^{N-k+j} \mathbf{J}_{d d} \widetilde{\mathbf{R}} \widetilde{\Lambda}_{Q}^{k-1-j} \widetilde{\mathbf{R}} \mathbf{J}_{d d}+ \\
(-1)^{j-1} \mathbf{J}_{d d} \widetilde{\mathbf{R}} \widetilde{\boldsymbol{\Lambda}}_{Q}^{k-1-j} \widetilde{\mathbf{R}} \mathbf{J}_{11}+ \\
(-1)^{N-1-k} \mathbf{J}_{11} \widetilde{\mathbf{R}} \widetilde{\boldsymbol{\Lambda}}_{Q}^{k-1-j} \widetilde{\mathbf{R}} \mathbf{J}_{d d}
\end{gathered}
$$

The first two terms of (19) are functions of $(k-j)$, while the last two are not. In the event that the first two terms of (19) are dominant, $c_{j k} \triangleq \underline{a}^{T} \Upsilon[j, k] \underline{b}$ will consist of a weighted sum of exponential terms, and will be a function of $(k-j)$. Indeed, we can see that the exponential terms in $c_{j k}$ will have the form $\left(\kappa_{i}^{\prime}\right)^{k-j}$. Consequently,

$$
L_{N, 2 b} \approx \sum_{n=1}^{d} \sum_{j<k} A_{n}\left(\kappa_{n}^{\prime}\right)^{k-j} \tilde{y}_{j} \tilde{y}_{k}
$$

for some constants $A_{n}, 1 \leq n \leq d$. The RHS of (20) for $1<$ $n<d$ looks like the sum of $(d-2)$ FE statistics, cf. (5). In the FE statistic, however, there are terms of the form $\tilde{y}_{j}^{2}$. Nonetheless, under certain conditions, they can be shown to be negligible [8]. For $n \in\{1, d\} \Longrightarrow\left|\kappa_{n}\right|=1$, and the corresponding terms in (20) can be generated by second-order polynomials in $\tilde{y}_{k}$. To summarize,

Proposition 4 For the LR of the class of random walks that we consider to be approximated by a bank of FE statistics under the conditions of low SNR and large N, the following conditions are necessary:

C1. The random walk $X_{i}$ is zero mean in steady-state. That is, $\lim _{i \rightarrow \infty} E\left[X_{i}\right]=0$.

C2. The coefficient $c_{j k}=\underline{a}^{T} \Upsilon \mathbf{\Upsilon}[j, k] \underline{b}$ is approximately a function of $(k-j)$.

C3. The squared terms $\tilde{y}_{j}^{2}$ in the FE statistics used to generate $L_{N, 2 b}$ in (20) are negligible compared to the cross-terms $\tilde{y}_{j} \tilde{y}_{k}$, $j \neq k$.

It is interesting to note that $L_{N, 2 b}$ is a function of the eigenvalues of $\mathbf{Q}$. Perhaps this is a general result that is true when $\mathbf{P}$ is an arbitrary probability transition matrix.

If conditions $\mathrm{C} 1-\mathrm{C} 3$ are satisfied, and

$$
c_{j k} \approx C^{\prime} \alpha^{k-j}
$$

for some $C^{\prime}$ and $\alpha$, then necessary conditions exist for the LRT of the random walk to be approximated by a single FE statistic.

Let us further investigate condition $\mathrm{C} 2$ and (21). Let $\widetilde{\mathbf{R}}=\left(\rho_{j k}\right)$. An asterisk in either the row or column index shall denote all valid values. For example, the notation $\rho_{1 *}$ refers to the first row of $\widetilde{\mathbf{R}}$, $\rho_{* d}$ refers to the last column of $\widetilde{\mathbf{R}}$, etc. For $\underline{\theta}, \underline{\tau} \in \mathbb{R}^{d}$, let $\underline{\theta} \odot \underline{\tau} \triangleq$ $\left[\theta_{1} \tau_{1}, \ldots, \theta_{d} \tau_{d}\right]^{T}$. Define $S: \mathbb{R}^{d} \rightarrow \mathbb{R}$ by $S(\underline{\theta})=\sum_{i=1}^{d} \theta_{i}$. Let $\underline{\kappa}^{\prime} \triangleq\left[\kappa_{1}^{\prime}, \ldots, \kappa_{d}^{\prime}\right]^{T}$ and use the notation that for $\underline{\theta} \in \mathbb{R}^{d}, \underline{\theta}^{<i>}=$

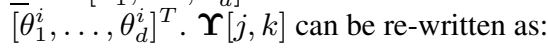

$$
\begin{gathered}
\Upsilon[j, k]=S\left(\left(\underline{\kappa}^{\prime}\right)^{<k-1-j>} \odot \rho_{1 *}^{T} \odot \rho_{* 1}\right) \mathbf{J}_{11}+ \\
(-1)^{N-k+j} S\left(\left(\underline{\kappa}^{\prime}\right)^{<k-1-j>} \odot \rho_{d *}^{T} \odot \rho_{* d}\right) \mathbf{J}_{d d}+ \\
(-1)^{j-1} S\left(\left(\underline{\kappa}^{\prime}\right)^{<k-1-j>} \odot \rho_{d *}^{T} \odot \rho_{* 1}\right) \mathbf{J}_{d 1}+ \\
(-1)^{N-1-k} S\left(\left(\underline{\kappa}^{\prime}\right)^{<k-1-j>} \odot \rho_{1 *}^{T} \odot \rho_{* d}\right) \mathbf{J}_{1 d}
\end{gathered}
$$

so that the dependence on $\kappa_{i}^{\prime}$ and $\rho_{j k}$ is clear.

We shall say that $c_{j k}$ is approximately a function of $(k-j)$ if the terms of the vectors $\left(\rho_{d *}^{T} \odot \rho_{* 1}\right)$ and $\left(\rho_{1 *}^{T} \odot \rho_{* d}\right)$ are negligible compared to $\left(\rho_{1 *}^{T} \odot \rho_{* 1}\right)$ and $\left(\rho_{d *}^{T} \odot \rho_{* d}\right)$. For example, the $l_{\infty}$ norm could be used, so that $c_{j k}$ is approximately a function of $(k-j)$ if $\left\|\rho_{1 *}^{T} \odot \rho_{* 1}\right\|_{\infty},\left\|\rho_{d *}^{T} \odot \rho_{* d}\right\|_{\infty} \gg\left\|\rho_{d *}^{T} \odot \rho_{* 1}\right\|_{\infty},\left\|\rho_{1 *}^{T} \odot \rho_{* d}\right\|_{\infty}$

Suppose that, in addition, there exists some $1<i<\left\lfloor\frac{d}{2}\right\rfloor$ such that

$$
\rho_{1 *}^{T} \odot \rho_{* 1} \approx C_{1} e_{i} \text { and } \rho_{d *}^{T} \odot \rho_{* d} \approx C_{2} e_{d+1-i}
$$

for some $C_{1}, C_{2} \in \mathbb{R}$, where the $e_{i}$ 's are the standard unit vectors in $\mathbb{R}^{d}$. Consequently,

$$
\begin{aligned}
c_{j k} & \approx C^{\prime} \alpha^{k-j} \\
\text { where: } C^{\prime} & =\left[C_{1} \underline{a}^{T} \mathbf{J}_{11} \underline{b}+(-1)^{N-1} C_{2} \underline{a}^{T} \mathbf{J}_{d d} \underline{b}\right]\left(\kappa_{i}^{\prime}\right)^{-1} \\
\alpha & =\kappa_{i}^{\prime}
\end{aligned}
$$

and the LRT can be approximated by a single FE detector. Specifically, if $\mathrm{C} 1$ is satisfied, then

$$
L_{N} \approx L_{N, 2 b} \approx C^{\prime} \kappa_{1}^{-2} \sum_{j<k} \alpha^{k-j} \tilde{y}_{j} \tilde{y}_{k}
$$

The statistic can be implemented by setting $\alpha_{\mathrm{LP}}=\alpha=\kappa_{i}^{\prime}$ in the FE detector, cf. (5), if C3 holds.

\section{SIMULATIONS}

Evaluation of the detectors was done using receiver operating characteristic (ROC) curves. Each ROC curve is a plot of the probability of false alarm $\left(P_{F}\right)$ vs. the probability of detection $\left(P_{D}\right)$, and was generated using 20 simulations. The averages were plotted for each curve, along with error bars of one standard deviation. The following detectors were evaluated: the unfiltered energy (UE) detector; the FE detector; the optimal random walk LRT (RW-LRT); and the matched filter bound (MF). When the signal $\underline{x} \triangleq\left[x_{0}, \ldots, x_{N-1}\right]^{T}$ is known to the receiver, the optimal LRT is the matched filter. It is unimplementable in reality, as the receiver does not have knowledge of $\underline{x}$. However, it provides an absolute upper bound when comparing the ROC curves.

In the simulations, $N=6 \times 10^{4}$ and $d=117$. Two random walk models were studied. The first had the probability transition matrix $\mathbf{P}_{1}$ given by

$$
\mathbf{P}_{1}(j, k)=\left\{\begin{array}{cc}
0.5 & 1<j<d, k=j \pm 1 \\
0 & \text { otherwise }
\end{array}\right.
$$


where $\mathbf{P}_{1}(j, k)$ denotes the $(j, k)$-th element of $\mathbf{P}_{1}$. The reflecting boundary conditions manifest as $\mathbf{P}_{1}(1,2)=\mathbf{P}_{1}(d, d-1)=1$; and the remaining elements of the first and last row of $\mathbf{P}_{1}$ are zero. The second random walk model studied had the probability transition matrix $\mathbf{P}_{2}$ given by

$$
\mathbf{P}_{2}(j, k)=\left\{\begin{array}{cc}
0.45 & j \in\left(1, \frac{d+1}{2}\right] \cup\left(\frac{3 d+1}{2}, d\right), k=j-1 \\
0.55 & j \in\left(1, \frac{d+1}{2}\right] \cup\left(\frac{3 d+1}{2}, d\right), k=j+1 \\
0.5 & j \in\left(\frac{d+1}{2}, \frac{3 d+1}{2}\right], k=j \pm 1
\end{array}\right.
$$

with the first and last row being identical to $\mathbf{P}_{1}$, i.e., the same reflecting boundary conditions.

The simulated ROC curve for the random walk model associated with $\mathbf{P}_{1}$ is given in Fig. 1 for $\mathrm{SNR}=-44.0 \mathrm{~dB}$. As $\max _{k}\left|\psi_{k} / \sigma\right| \approx$ $1.76 \times 10^{-2}$, the detection problem is in the regime of low SNR. The performance of the FE detector is approximately the same as

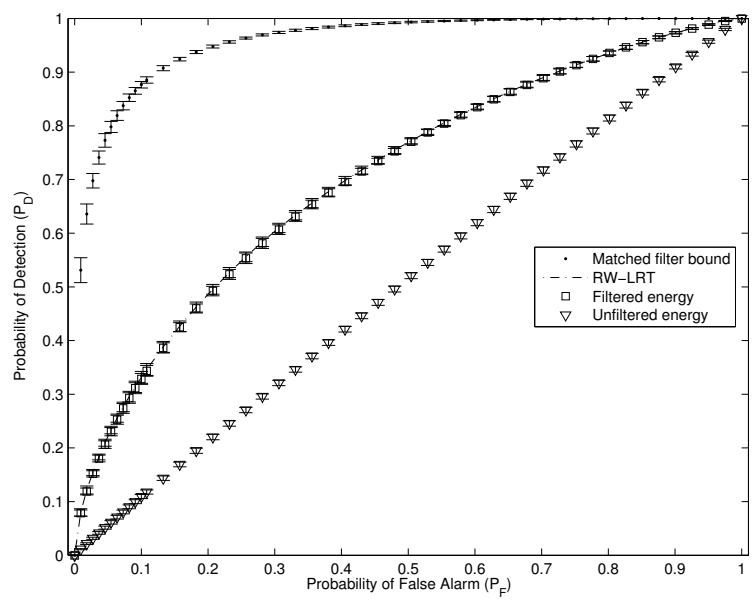

Fig. 1. Simulated ROC curve for the random walk model with probability transition matrix $\mathbf{P}_{1}$ at $\mathrm{SNR}=-44.0 \mathrm{~dB}$.

the optimal RW-LRT. The MF bound has the best performance, as was expected. The UE detector has the worst performance, and is no better than flipping a fair coin. Conditions C1, (23), (24), and $\mathrm{C} 3$ hold for the first random walk model. Consequently, the necessary conditions for the RW-LRT to be approximated by a single FE detector are satisfied. The simulated ROC curve for the random walk model associated with $\mathbf{P}_{2}$ is given in Fig. 2 for $\mathrm{SNR}=-2.50$ $\mathrm{dB}$. As before, the MF bound has the best performance, and the UE detector the worst. For the second random walk model, however, the FE detector performs noticeably poorer than the RW-LRT. However, condition $\mathrm{C} 1$ is not satisfied, and so we would not expect a single FE statistic to well approximate the RW-LRT. For the second model, $\max _{k}\left|\psi_{k} / \sigma\right| \approx 9.88 \times 10^{-3}$. The detection problem is in the regime of low SNR, even though the SNR is higher than in the previous example.

\section{CONCLUSIONS}

In this paper, we presented a closed form expression for the LR of a Markov signal in AWGN. The LRT is the optimal test, in the Neyman-Pearson sense, for the binary hypothesis problem. Necessary conditions were obtained for the LRT of a class of random walk processes to be approximated by a bank of filtered energy detectors

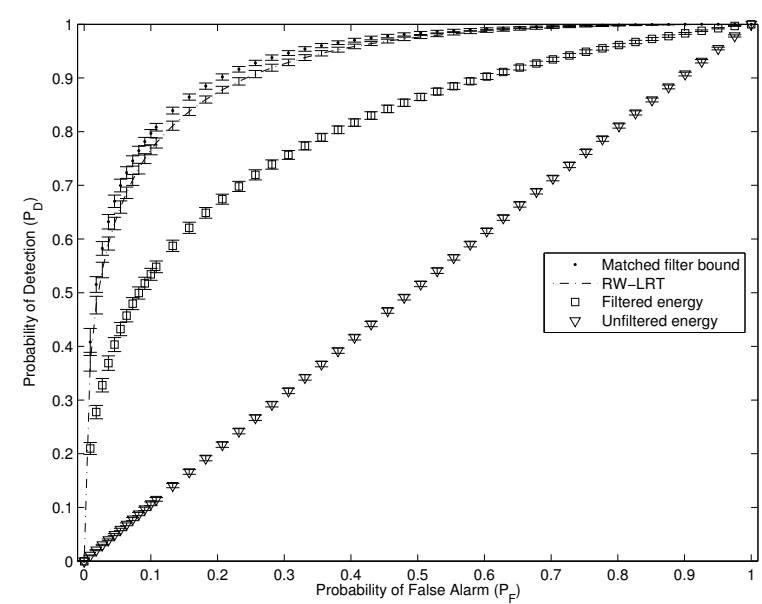

Fig. 2. Simulated ROC curve for the random walk model with probability transition matrix $\mathbf{P}_{2}$ at $\mathrm{SNR}=-2.50 \mathrm{~dB}$.

under the regime of low SNR and long observation time. An additional condition is required for the LRT to be approximated by a single filtered energy detector. A simulation study produced results that were consistent with the analysis.

\section{REFERENCES}

[1] M. Ting, A. O. Hero, D. Rugar, C.-Y. Yip, and J. A. Fessler, "Near optimal signal detection for finite state Markov signals with application to magnetic resonance force microscopy," IEEE Trans. Signal Processing, to appear.

[2] D. Rugar, R. Budakian, H. J. Mamin, and B. W. Chui, "Single spin detection by magnetic resonance force microscopy," Nature, vol. 430, no. 6997, pp. 329-332, 2004.

[3] M.-H. Zhang and Q.-S. Cheng, "Gaussian mixture modelling to detect random walks in capital markets," Mathematical and Computer Modelling, vol. 38, pp. 503-508, 2003.

[4] Y.-M. Wang and N. R. Sheeley Jr., "On the fluctuating component of the sun's large-scale magnetic field," The Astrophysical Journal, vol. 590, pp. 1111-1120, 2003.

[5] S. K. Mitra, Digital Signal Processing: A Computer-Based Approach, McGraw-Hill, New York, second edition, 2001.

[6] H. L. Van Trees, Detection, Estimation, and Modulation Theory, vol. 1, Wiley, New York, 1968.

[7] L. L. Scharf and L. W. Nolte, "Likelihood ratios for sequential hypothesis testing on Markov sequences," IEEE Trans. Inform. Theory, vol. IT-23, no. 1, pp. 101-109, 1977.

[8] M. Ting, Signal processing for magnetic resonance force microscopy, Ph.D. thesis proposal report, University of Michigan, 2005.

[9] D. K. Ross, "Eigenvalues of a tri-diagonal matrix: problem 80-4,” SIAM Review, vol. 23, no. 1, pp. 112-113, 1981.

[10] S. Karlin and H. M. Taylor, A second course in stochastic processes, Academic Press, New York, 1981. 